\title{
Swot Analysis Of Software Quality Metrics For Global Software Development: A Systematic Literature Review Protocol
}

\author{
Sadia Rehman ${ }^{1,2}$, Siffat Ullah Khan ${ }^{1,2,3}$ \\ ${ }^{1}$ Software Engineering Research Group (SERG_UOM), \\ ${ }^{2}$ Department of Computer Science and IT, \\ ${ }^{3}$ Department of Software Engineering, \\ University of Malakand, Khyber Pakhtunkhwa, Pakistan.
}

\begin{abstract}
CONTEXT - Global Software Development (GSD) is a modern software engineering paradigm adopted by many client organisations in developed countries to get high quality product at low cost in low wage countries. Production of high quality software is considered as one of the key factor in the rapid growth of GSD. However GSD projects have put new challenges to practitioners and researchers. In order to address these challenges Software Quality Metrics (SQMs) are frequently used in organisations to fabricate high quality products.

OBJECTIVE - The objective of this SLR protocol is to identify and assess strengths and weaknesses of the existing SQMs used in GSD to assist vendor organisations in choosing appropriate SQMs for measuring software quality.

METHOD - Systematic Literature Review (SLR) will be used for the identification of the existing SQMs in GSD. $S L R$ is based on a structured protocol, and is therefore, different from ordinary review.

EXPECTED OUTCOME - We have developed the SLR protocol and are currently in process of its implementation. The expected outcomes of this review will be the identification of different SQMs for GSD along with their SWOT analysis to assist vendor organisations in choosing appropriate SQMs at the right time to produce a high quality product.
\end{abstract}

Keywords - Software Quality Metrics, Global Software Development, Systematic Literature Review Protocol

\section{Introduction}

Global Software Development (GSD) has become the fashion of software industry from the last two decades. GSD is the fabrication of software product in vendor's countries to get high quality product at affordable cost for clients[1]. To survive in the global market, oragnisations endeavor their best to fabricate high quality product as the quest is now for the high quality.

GSD is leading the software industry in instance. GSD is an increasing fashion of the industry so we can say that the future software industry will be a global software industry in reality. Globalization makes the software development more complex than a standalone software development, that's why organisation engage in GSD have more challenges to face, like timely delivery of products, skilled human resources, sharing of knowledge and quality of product and service, contract management, hidden costs[1]. According to IEEE "Software quality is the degree to which a system, component, or process meets specified requirements and meets customer's needs or expectations"[2]. Quality of software often expresses in its attributes which can be further subdivided into other attributes such as efficiency, maintainability, testability, flexibility, interface facility, reusability, and transferability[3]. These internal and external attributes group together to define the quality of a software [4].

Measurement is a process that helps the organisations to evaluate their products. Measurement serves to control and predicate things [5]. In software industry the measurement culture is not innovative, measurement gives the quantitative values which are easy to interpret [6]. Horst Zuse [7] defined that researchers are active in the area software measurement since more than thirty years, this area is known as software metrics[8]."Measurement is the process by which numbers or symbols are assigned to attributes of entities in the real world so as to describe such entities according to clearly defined rules". Software Quality Metrics (SQMs) are used in software industry to enhance the quality and stay away from all possible risks that organisations have to face in software development process [9]. "Software quality metrics are a subset of software metrics that focus on the quality aspects of the product, process, and project. In general, software quality metrics are more closely associated with process and product metrics than with project metrics"[10]. The use of metrics can be more productive when organizations choose the appropriate metrics according to their need at the right time.

The main objective of this research is to study thoroughly the existing literature and gain intensive understanding of SQMs for GSD through a Systematic literature review (SLR). The following two research questions (RQs) were formulated to be answered through SLR. 
$\mathrm{RQ1}$. What are the different quality attributes, as identified in the literature, that affect software quality in GSD? RQ2. What are the existing software quality metrics (SQMs) in GSD, as identified in the literature?

RQ2.1. What is SWOT analysis of existing SQMs for GSD?

RQ3. What are the different quality attributes, as identified in the real world practice, which affect software quality in GSD?

RQ4. What are different the software quality metrics, as identified in the real world practice, which affect software quality in GSD?

\section{Background}

Software industry is using software metrics since when "source lines of code" or SLOC was developed for quantifying the output of a software project [11]. Vendor organisations use metrics to improve its quality by measuring its capabilities and efficiencies. Using software metrics in GSD plays a vital role to avert on hand and future risks and to improve its software quality [12]. The purpose of software measurement is to quantify all attributes of quality and predict the future quality of the software [13]. The use of appropriate software metrics at right time helps the organisations to achieve their required and expected outcomes. The use of SQMs facilitate the organisations to get both short and long term advantages by introducing high quality product to the global market [14].

\subsection{Published Literature on Software Quality and Software Quality Metrics}

The concept of software quality is the core of software development life cycle in GSD. Organisations have more challenges to meet the expectations and requirements of clients. Quality is being gauged by measuring its internal and external attributes [15]. The measurement process is done with the help of Software Quality Metrics. A number of researchers have worked to address various issues in this domain. e.g.

- "Software quality is the extent to which an industry-defined set of desirable features are incorporated into a product so as to enhance its lifetime performance"[16].

- ISO standards of quality are being adapted by organisations in GSD to excel their performance. ISO/ICE 9126 quality model have various internal and external quality factors [17].

- Kitchenham defined quality as "Quality is a complex concept. Because it means different things to different people, it is highly context-dependent. Just as there is no one automobile to satisfy everyone's needs, so too there is no universal definition of quality. Thus, there can be no single, simple measure of software quality acceptable to everyone. To assess or improve software quality in your organisation, you must define the aspects of quality in which you are interested, and then decide how you are going to measure them"[18].

- Dr. Deepshikha Jamwal discussed different quality models (McCall's Quality Model, Boehm's Quality Model, Dromey's Quality Model, FURPS Quality Model, ISO 9126 Quality Model.) and concluded that only "reliability" is the common attribute in all models. A criteria has been defined based on some questions in order to choose quality model for any organisation that will save organisation's time [19].

- SQMs for GSD assist to have control over quality as according to Tom DeMacrio "You cannot control what you cannot measure. Measurement is the prerequisite to management control" [5]

- In order to avoid software crisis effective software management is required that can be facilitated by the use of software metrics [9]

- Barbara Kitchenham has conducted a survey to describe advancement in software metrics research. The study assesses 103 papers published between 2000 and 2005. She has suggested that researchers in software metrics domain need to refine their empirical methodology to answer empirical questions [20].

GSD induces the organisations to invest more cost and efforts to get high software quality by selecting appropriate SQMs according to their requirements at right time. The use of software metrics becomes ineffective and extra load on organisations when metrics do not observe the goals of organisations [21].

This research will help the organisations to choose appropriate SQMs for GSD at right time to fabricate high quality product. The outcomes of this research will be SWOT Analysis of currently used SQMS in software industry. In order to make the work more reliable we are using systematic review approach. A systematic literature review is a way of discovering, assessing and inferring all available research relevant to a particular research question, or topic area [22]. Currently we are presenting the planning phase of the SLR which is the first step of SLR.

\section{Systematic Literature Review Protocol For SWOT Analysis Of Software Quality Metrics For Global Software Development}

A systematic literature review is a way of discovering, assessing and inferring all available research relevant to a particular research question, or topic area [22]. This paper illustrates the SLR protocol on SQMs for GSD. Formally SLR has three phases which are as follows: 
1. Planning the review

2. Conducting the review

3. Reporting the review

The expected outcomes of this review will be the identification of different SQMs for GSD along with their SWOT analysis to assist vendor organizations to select appropriate SQMs at the right time to get high quality product.

\subsection{Construction of Search Terms}

Following details will be supportive in constructing search terms.

- Population: Software quality in Global Software Development(GSD), quality metrics in GSD

- Intervention: Quality attributes, characteristics, factors, parameters, software quality measures

- Outcomes of relevance: Quality Software

- Experimental Design: Empirical studies, theoretical studies, case studies, experts' opinions

RQ1:

Example of research questions containing the above details are:

[What are the different quality attributes/ factors] INTERVENTION

that affect

[Software quality]

\section{OUTCOME OF RELIVENCE}

in

[Global Software Development]

\section{POPULATION}

\subsection{Search Strategy}

\section{2 .1Trial Search}

A trial search was conducted using the following search string on

- IEEE Explore (http://ieeexplore.ieee.org)

- Google Scholar(scholar.google.com)

- Emerald digital library(http://www.emeraldinsight.com)

\subsubsection{Trail Search}

("Software quality" OR "software quality assurance" OR "software quality management") AND (factors OR attributes OR characteristics) AND ("software quality metrics" OR "software quality measuring tools" OR "software quality assessment tool") AND ("Global software development" OR GSD OR "offshore software outsourcing" OR "offshore software development outsourcing" OR OSDO))

\begin{tabular}{|c|c|c|c|c|}
\hline $\begin{array}{c}\text { S. } \\
\text { No }\end{array}$ & $\begin{array}{c}\text { Digital } \\
\text { Library }\end{array}$ & $\begin{array}{c}\text { Total result } \\
\text { Displayed }\end{array}$ & $\begin{array}{c}\text { No of Relevant } \\
\text { Papers }\end{array}$ & $\begin{array}{c}\text { Date of } \\
\text { Search }\end{array}$ \\
\hline $\mathbf{1}$ & IEEE Explorer & 322 & $\mathbf{1 0 0}$ & 6-June-2012 \\
\hline $\mathbf{2}$ & $\begin{array}{l}\text { Google } \\
\text { Scholar }\end{array}$ & 780 & $\mathbf{5 7 6}$ & 6-June-2012 \\
\hline $\mathbf{3}$ & Emerald & $\mathbf{3 1 5}$ & $\mathbf{9 0}$ & 6-June-2012 \\
\hline
\end{tabular}

Table 1: Trial Search Results

This search string is used as a guide for the development and validation of major search terms.

\subsection{Identifying Search Terms for SQMs for GSD}

The search strategy for the SLR is a plan to:

a. Construct search terms by distinguishing population, intervention and outcome.

b. Discover the alternative spellings and synonyms.

c. Verify the key words in any relevant paper.

d. Use Boolean Operators.

e. Integrate the search string into a summarized form, if required.

The fifth step is included from the SLR study [23].

Result for a)

RQ1: Software quality, attributes, Global software development

RQ2: Software quality metrics, Global software development 
Result for b)

RQ1:

Software quality: ("Software quality" OR "software quality management" OR "software quality assurance" OR "Application quality" OR SQA OR "total quality management” OR "software standard” OR SQM OR "software rank" OR "software ability" OR "software caliber")

Attributes: (Characteristics OR aspects OR factors OR features OR components OR parameters OR drivers OR motivators)

GSD: ("offshore software outsourcing" OR "information systems outsourcing" OR "information technology outsourcing” OR "IS outsourcing” OR "IT outsourcing” OR “CBIS outsourcing” OR "computerbased information systems outsourcing" OR "software-contracting-out" OR "distributed software development" OR "multi-site software development" OR "global software development" OR "GSD” OR "offshore software development outsourcing" OR OSDO)

RQ2:

Software quality: ("Software quality" OR "software quality management" OR "software quality assurance" OR "Application quality" OR SQA OR "total quality management” OR "software standard” OR SQM OR "software rank" OR "software ability" OR "software caliber")

Metrics: (Metrics OR “measuring tool" OR “assessment tool" OR "evaluation tool" OR "quantification tool" OR measure)

GSD: ("offshore software outsourcing" OR "information systems outsourcing" OR "information technology outsourcing" OR "IS outsourcing” OR "IT outsourcing” OR "CBIS outsourcing" OR "computerbased information systems outsourcing" OR "software contracting-out" OR "distributed software development" OR "multi-site software development" OR "global software development" OR "GSD" OR "offshore software development outsourcing" OR OSDO)

\section{Result for c)}

development

Software quality, software quality attributes, software quality metrics, and Global software

Result for d)

RQ1:

("Software quality factors" OR "Software quality" OR "Software standard" OR "Software Quality Management" OR SQM OR "Software Quality Assurance" OR "Application quality" OR SQA OR "Software rank" OR "Software ability" OR "software caliber") AND (Characteristics OR aspects OR factors OR features OR components OR parameters OR drivers OR motivators) AND ("Global software development" OR GSD OR "Offshore software outsourcing" OR "Information systems outsourcing" OR "Information technology outsourcing" OR "IS outsourcing" OR "IT outsourcing" OR "CBIS outsourcing" OR "Computer-based information systems outsourcing" OR "Distributed Software Development" OR "Multi-site Software Development" OR OSDO))

RQ2:

("Software quality" OR "Software standard" OR "Software Quality Management" OR SQM OR "Software Quality Assurance" OR "Application quality" OR SQA OR "Software rank" OR "Software ability" OR "software caliber" OR "total quality management") AND (Metrics OR "measuring tool" OR "assessment tool" OR "quantification tool" OR measure OR "evaluation tool") AND ("Global software development" OR GSD OR "Offshore software outsourcing" OR "Information systems outsourcing" OR "Information technology outsourcing" OR "IS outsourcing" OR "IT outsourcing" OR "CBIS outsourcing" OR "Computer-based information systems outsourcing" OR "Distributed Software Development" OR "Multi-site Software Development" OR OSDO))

Result for e)

As the strings successfully retrieved relevant papers therefore no result for e.

\subsection{Search Terms Breakup}

We break down the above mention search strings as some databases do not allow length search strings, for this reason we will do the following steps

- Fragmented search string in small substrings

- Execute separate search for these strings

- Summaries to eliminate redundancy

- In IEEEXplore and ScienceDirect Digital libraries, search string will be used with a technique that it should be put in the pane, large rectangular box, instead of the small sized text-boxes furnished in the advanced search of the library. 


\section{Substrings for RQ1}

\section{String 1}

("Software quality factors" OR "Software quality" OR "Software rank" OR "Software ability") AND (Characteristics OR aspects) AND ("Global software development" OR GSD OR "Offshore software outsourcing" OR "IS outsourcing"))

String 2

(SQM OR "Software Quality Assurance" OR "Application quality") AND (factors OR features OR parameters OR motivators) AND ("IT outsourcing" OR "CBIS outsourcing" OR "Computer-based information systems outsourcing" OR "Distributed Software Development")

String 3

("Software standard" OR SQA OR "Software Quality Management" OR "software caliber") AND (components OR drivers) AND ("Information systems outsourcing" OR "Information technology outsourcing" OR "Multi-site Software Development" OR OSDO))

\section{Substrings for RQ2}

String 1

("Software quality" OR "Software standard" OR "Software Quality Management") AND (Metrics OR "measuring tool") AND ("Global software development" OR GSD OR "Offshore software outsourcing" OR "Information systems outsourcing" OR "IT outsourcing"))

String 2

(SQMs OR "Software Quality Assurance" OR "Application quality" OR "Software rank") AND ("assessment tool" OR "quantification tool") AND ("Information technology outsourcing" OR "IS outsourcing" OR "CBIS outsourcing" OR "Distributed Software Development"))

String 3

(SQA OR "Software ability" OR "software caliber" OR "total quality management") AND (measure OR "evaluation tool") AND ("Computer-based information systems outsourcing" OR "Multi-site Software Development" OR GSD)

\subsection{Perform Search for Relevant Studies}

Resources to be searched

- ACM Portal (http://dl.acm.org)

- ScienceDirect (www.sciencedirect.com )

- CiteSeer Digital Library (www.citeseer.ist.psu.edu)

- Google Scholar (www.scholar.google.com )

- IEEExplore (http://ieeexplore.ieee.org )

- Emerald (http://www.emeraldinsight.com )

- SpringerLink (www.springerlink.com )

\subsection{Search Constraints and Validation}

We are searching through literature as follow.

- Search for all relevant literature without any time (years) boundary/limit

- Initially few relevant papers were found through major search terms

- Prior to undertake the review, these relevant papers will be used for the validation of the search strings.

\subsection{Search Documentation}

The candidate papers will be stored in the form of a table with keeping following records:

(S. No, Name of Database, Search Strategy, Search Phase, Date of Search, Years Covered, No of Publication Found, Initial Selection Decision, Final Selection Decision

\subsection{Search Result Management}

The records of all the candidate papers from the digital libraries are kept in a directory. Record of each digital library is stored in separate directory, where each page is saved as.html page.

\subsection{Inclusion Criteria}

\section{Publication Selection}

The inclusion criteria we used to authenticate which piece of literature found by the search string(s) will be used for the data extraction. We will only consider papers related to Software Quality Metrics with focus on GSD that are written in English. The criteria are listed below.

- Studies that describe SQMs for global software development

- Studies that describe different attributes of software quality in GSD

- Studies that describe exiting SQMs in GSD 
- Studies that describe motivation/demotivation in SQMs for GSD

- Studies that describe software measurement and software quality in GSD

- Studies that describe strengths and weaknesses of SQMs for GSD

\subsection{Exclusion Criteria}

The exclusion criteria are listed as follow

- Studies that are not relevant to the research questions

- Studies that don't describe attributes of software quality and software quality metrics in GSD

- Studies that don't describe SQMs for GSD

- Studies that don't describe software measurement and software quality in GSD

- Studies other than SQMs

\subsection{Selecting Primary Sources}

Initial selection of the primary sources will be executed by reviewing the title, keywords and abstract. The inclusion/exclusion criteria will be checked against each selected paper after reviewing its full text. The record of inclusion/exclusion decision regarding each primary source will be sustained properly with suitable justification.

\begin{tabular}{|c|c|c|c|c|c|c|}
\hline $\begin{array}{l}\text { S. } \\
\text { No }\end{array}$ & $\begin{array}{l}\text { Digital } \\
\text { library }\end{array}$ & $\begin{array}{c}\text { Total Papers for } \\
\text { RQ1 }\end{array}$ & $\begin{array}{c}\text { Primary } \\
\text { Selection for RQ } \\
1\end{array}$ & $\begin{array}{l}\text { Total Papers } \\
\text { for RQ2 }\end{array}$ & $\begin{array}{l}\text { Primary Selection } \\
\text { for } R Q 2\end{array}$ & $\begin{array}{l}\text { Date of } \\
\text { Search }\end{array}$ \\
\hline 1 & $\mathrm{ACM}$ & 441 & 47 & 400 & 43 & $\begin{array}{l}\text { 5-14 June- } \\
2012\end{array}$ \\
\hline 2 & $\begin{array}{l}\text { Google } \\
\text { Scholar }\end{array}$ & 1277 & 710 & 747 & 360 & $\begin{array}{l}\text { 5-14 June- } \\
2012\end{array}$ \\
\hline 3 & Cite Seer & $1,477(500)$ & 46 & $701(500)$ & 64 & $\begin{array}{l}\text { 5-14 June- } \\
2012\end{array}$ \\
\hline 4 & SpringerLink & 114 & 19 & 74 & 18 & $\begin{array}{l}\text { 5-14 June- } \\
2012\end{array}$ \\
\hline 5 & Emerald & 68 & 7 & 470 & 50 & $\begin{array}{l}\text { 5-14 June- } \\
2012\end{array}$ \\
\hline 6 & IEEExplore & 529 & 132 & 218 & 39 & $\begin{array}{l}\text { 5-14 June- } \\
2012\end{array}$ \\
\hline \multirow[t]{2}{*}{7} & Science Direct & 111 & 29 & 113 & 41 & $\begin{array}{l}\text { 5-14 June- } \\
2012\end{array}$ \\
\hline & & Total : 3040 & Total :990 & Total : 2723 & Total : 615 & $\begin{array}{l}\text { 5-14 June- } \\
2012\end{array}$ \\
\hline
\end{tabular}

\section{Publication Quality Assessment}

The measurement of quality is executed after final selection of publications. The quality of publications is evaluated in parallel at the time of data extraction. The quality checklists include the following questions:

- Is it clear how quality attributes were identified in GSD?

- Is it clear how quality attributes were measured/evaluated in GSD?

- Is it clear how the use of SQMs for GSD is essential?

Each of the above factors will be marked as 'YES' or 'NO' or 'Partial' or 'N.A'.

\section{Data Extraction Strategy}

The following subsections are considered in the data extraction strategy

\subsection{Primary Study Data}

The rationale of the study is to accumulate the data, from the finally selected publications, which is concerned on satisfying the research questions for the review. The following data will be extracted from each publication Title, Authors, Journal/Conference title, etc, background information and information related to research questions.

\subsection{Data Extraction Process}

The review will be attempted by a single researcher, who will be responsible for the data extraction. A secondary reviewer will be approached for guidance in case of an issue concerning the data extraction. The inter-rater reliability test will be executed after the data extraction process by the primary reviewer. 


\subsection{Data Storage}

The summarized data for each publication will be kept as a Microsoft Word/SPSS document and will be stored electronically.

\section{Data Synthesis}

Due to two research questions, the synthesis will be categorized into two parts. The extracted data for RQ1 will be stored in a table having columns (S.No, Quality Attributes, Frequency, Percentage). For the RQ2; the table will have the following columns (S. No, software quality metrics, Frequency, Percentages).

\section{Acknowledgements}

We are obliged to Software Engineering Research Group at University of Malakand (SERG_UOM) in general and to Muhammad Ilyas Azeem in particular for the review and their valuable comments in validation process of the protocol.

\section{References}

[1] S. U. Khan, "Software outsourcing vendors' readiness model (SOVRM)," Keele University UK, 2010.

[2] IEEE, "IEEE Std 610.12-1990-IEEE Standards Glossary of Software Engineering Terminology, IEEE Computer Society," IEEE Std 610.12-1990, 1991.

[3] S. R. Pressman, Software Engineering : A practitioner's Approach, Sixth Edition ed.

[4] N. Fenton, "Software Measurement: A Necessary Scientific Basis," IEEE TRANSACTIONS ON SOFTWARE ENGINEERING, NO. 3. MARCH 1994, vol. VOL. 20, 1994.

[5] T. DeMacri, "Controlling Software Projects," 1982.

[6] "A Software Metrics Primer," 2007

[7] S. L. P. Fenton Norman E, Software Metrics: a Rigorous and Practical Approach, 2nd Edition ed, 1997.

[8] H. Zuse, Bollmann-Sdorra, and Peter, "Measurement Theory and Software Measures," presented at Proceedings of the BCSFACS Workshop on Formal Aspects of Measurement, London, 1991.

[9] E. M. Everald, "Software Metrics SEI Curriculum Module SEI-CM-12-1.1," Seattle University Seattle, Washington, Washington December 1988

[10] L. L. Westfall, "Twelve Step to Useful Software Metrics," presented at Proceedings of the Seventeenth Annual Pacific Northwest Software Quality Conference, 2005.

[11] J. Capers, "STRENGTHS AND WEAKNESSES OF SOFTWARE METRICS," Software Productivity Research LLC, 2006.

[12] M. Xenos, "Software Metrics and Measuremnts," Encylopedia of E-Commerce,E-Government and Mobile Commerce, pp. 8, 2006.

[13] J. Magne, "Software quality measurement."

[14] S. F.Norman "Knowledge Requirements for Software Quality Measurement," Empricial Software Engineering, 2001.

[15] Z. Dave, "Measuring Software Product Quality: the ISO 25000 Series and CMMI," European SEPG, 2004.

[16] R. Fitzpatrick, "Software Quality:Definitions and Strategic Issues," in School of Computing Report. County of Staffordshire, UK: Staffordshire University, 1996.

[17] ISO, "ISO 8402," 1994.

[18] B. Kitchenham and S. L. Pfleeger, "Software Quality: The Elusive Target," IEEE SOFTWARE 0740-7459/96/\$05.00, vol. Vol. 13, No. 1, 1996.

[19] S. Malathi and S. Dr S, "Analysis of Size Metrics and Effort Performance Criterion in Software Cost Estimation," Indian Journal of Computer Science and Engineering (IJCSE), vol. 3, 2012.

[20] B. Kitchenham, "What's up with software metrics? - A preliminary mapping study," The Journal of Systems and Software 83 (2010) 37-51, pp. 37-51, 2009.

[21] H. Douglas, "The Darker Side of Metrics," 2000.

[22] B. Kitchenham, "Guidelines for performing Systematic Literature Reviews in Software Engineering," Software Engineering Group, School of Computer Science and Mathematics and Department of Computer Science University of Durham, ST5 5BG,Durham,, EBSE Technical Report 9 July 2007.

[23] M. I. Azeem and S. U. Khan, "Intercultural Challenges in Offshore Software Development Outsourcing Relationships: A Systematic Literature Review Protocol," presented at 5th Malaysian Conference in Software Engineering (MySEC), Johor Bahru, Malaysia, 2011 